\title{
Complementary Therapies for Chronic Pain Management
}

\section{Jane Hart, M.D.}

$P$ ain is one of the most common medical conditions that people report in visits to physicians. Conditions causing pain may be acute or chronic, and the pain they cause can range from mild and intermittent to severe and unrelenting. Common causes of chronic pain include conditions of the back, arthritis, headache, and cancer.

It is estimated that $9 \%$ of the adult population of the United States suffers from moderate to severe non-cancer-related chronic pain. ${ }^{1}$ In addition, the American Cancer Society (ACS) estimates that $30 \%$ of patients with newly diagnosed cancer, $30 \%-50 \%$ of patients undergoing treatment for cancer, and $70 \%-90 \%$ of those with advanced malignant disease experience pain. ${ }^{2}$

Anyone who has suffered from chronic pain knows how exhausting and frustrating the experience can be. People with chronic pain report that it impairs their ability to work, exercise, socialize, perform activities of daily living, sleep, engage in sex, and relax. ${ }^{3}$ According to the American Academy of Pain Medicine (AAPM), "the annual cost of chronic pain in the United States, including health care expenses, lost income, and lost productivity is estimated to be $\$ 100$ billion." 4

Chronic pain may also have physiologic effects that go beyond what one might anticipate. A recent study by Baliki and colleagues, published in the Journal of Neuroscience, suggests that chronic pain may actually alter the functional connectivity between cortical connections in the brain-affecting brain function and specifically cognitive and behavioral functioning. ${ }^{5}$

Unfortunately, chronic pain is often inadequately addressed both by patients it affects and by medical practitioners. According to the AAPM, more than $50 \%$ of people with chronic pain say they have little or no control over their pain, ${ }^{4}$ and the ACS states that people with cancer often have pain that is untreated, undertreated, or improperly treated.

The reasons for inadequate pain management are varied and may include a lack of knowledge about how to manage pain, a lack of follow-up, a lack of diligence in trying different strategies if a first one does not work, and lack of a multimodal and integrative approach to managing pain. When pain is not immediately brought under control after a visit to a physician, many patients believe there is nothing more that the physician can do and do not follow-up by continuing to seek care. People with chronic pain also may delay seeking the help of a physician until 6 months after the pain starts. All of these factors interfere with the ability to address pain properly.

It is important to remember that relief of chronic pain rarely occurs overnight; the healing process takes time, commitment, and an investment by the individual experiencing the pain as well as by the medical and complementary and alternative medicine (CAM) practitioners treating it. Success in treating chronic pain is increased by the use of an integrative approach.

\section{Defining Chronic Pain}

Chronic nonmalignant pain is defined by the American Pain Society (APS) as "[g]enerally considered to be pain that lasts more than 6 months, is ongoing, is due to non-life-threatening causes, has not responded to current available treatment methods, and may continue for the remainder of the person's life. People with chronic pain may also experience episodes of acute pain." ${ }^{6}$

The APS defines cancer pain as "[a] cute, chronic, or intermittent [pain that] often has a definable etiology, usually related to tumor recurrence or treatment." 6

According to the Chronic Pain in America Survey, ${ }^{3}$ most people who suffer from chronic pain are: (1) currently in pain; (2) have experienced pain for more than 5 years; (3) have flare-ups of their pain; (4) rate their pain as moderate (6 or 7 on a scale of 10); and have arthritic or back pain.

\section{The Role of Complementary Therapies in Treating Chronic Pain}

Unfortunately, common analgesic agents do not always fully relieve chronic pain. Integrating complementary therapies into a conventional medical plan can help ease chronic pain and reduce the need for medication.

Chronic pain is a motivator for the use of CAM therapies. Integrating CAM therapies into a conventional treatment plan may yield better results.

Complementary therapies as adjuncts to a conventional treatment plan may help: (1) reduce or eliminate chronic pain; (2) reduce the need for medications; (3) improve mood and affect; (4) 
enhance a sense of well-being; (5) reduce stress and stress-related visits to physicians; (6) improve functionality and the ability to perform activities of daily living; and (7) provide the patient with a greater sense of control over his or her life.

Conditions marked by chronic pain are the number one reason why adults use CAM therapies, according to the National Center for Complementary and Alternative Medicine's 2004 survey on patterns of use of complementary/alternative medicine. ${ }^{7}$ In this survey, back pain, neck pain, joint pain, and arthritis were the most common pain-related conditions ${ }^{8}$ prompting the use of CAM.

Additionally, pediatricians are most likely to refer children for CAM therapy for chronic problems such as headaches and pain management. ${ }^{8}$ Children are also most likely to be referred for CAM therapies for chronic problems in which pain may be a central issue, such as inflammatory bowel disease or cancer. ${ }^{9}$

When children in a pediatric chronic pain clinic were surveyed about their interest in CAM and specific therapies, ${ }^{9}$ more than $60 \%$ of the children were interested in CAM therapies. They were most interested in biofeedback, yoga, and hypnosis and least interested in art therapy and energy healing. Children in the survey preferred noninvasive treatments that provided relaxation and somatic control. A longer duration of pain and impaired functioning were predictors of greater interest in trying CAM therapies. ${ }^{9}$

A study of interest in CAM among adults with chronic back pain found that they were most interested in massage as a complementary therapy for their pain, and were also interested in acupuncture and $t^{\prime}$ ai chi despite knowing very little about these therapies. ${ }^{10}$

The following sections cover a sampling of common complementary therapies used to help manage chronic pain. The sections do not cover all of the available evidence for CAM and chronic pain, nor do they cover every chronic pain syndrome or discuss the use of dietary supplements and herbs for chronic pain, but are instead intended to provide an overview of chronic pain with insights from the current literature.

\section{Acupuncture}

Research has shown that acupuncture may reduce pain through a variety of mechanisms including stimulation of the nervous system and the release of hormones that regulate pain, as well as by reducing activation of areas in the brain associated with pain. ${ }^{11}$ The exact mechanism by which acupuncture affects pain is not known.

Nevertheless, many reports describe physician recommendations of acupuncture for chronic pain, and patients' statements of benefits from acupuncture for back pain, arthritic pain, headaches, temporomandibular joint (TMJ) syndrome, cancer pain, fibromyalgia, and other types of chronic pain.

A retrospective study of people seeking acupuncture at the Stanford University Complementary Medicine Clinic (Stanford, California) found that the majority $(79 \%)$ did so for conditions marked by chronic pain including back pain, degenerative disk disease, and fibromyalgia. ${ }^{12}$ Most referrals to the clinic came from physiatrists / orthopedists and pain-management specialists.
One study of the role of acupuncture in treating chronic headaches randomly assigned 401 primary-care patients with headache (predominantly migraine) disorders to receive 12 acupuncture treatments over a 3-month period or to have no acupuncture. ${ }^{13}$ The researchers found that the acupuncture group made $25 \%$ fewer visits to physicians, used 15\% less medication,

\section{A longer duration of pain and impaired functioning were predictors of greater interest in trying CAM therapies.}

and had $15 \%$ fewer sick days than did the group not given acupuncture. The scientists concluded that acupuncture may provide lasting beneficial effects for people with headache disorders, and, particularly, patients with migraine headaches.

The use of acupuncture for chronic musculoskeletal and arthritic pain is reported to be common and may be more successful as an adjunct to conventional treatment. ${ }^{14}$

In a study of 241 adults who were randomly assigned to receive usual care or 10 acupuncture treatments for chronic low-back pain, ${ }^{15}$ weak evidence was found in pain scores for a beneficial effect of acupuncture at 12 months of follow-up and stronger evidence was found for a benefit at 24 months of follow-up as opposed to usual care. The authors concluded that referral to a qualified traditional acupuncturist for a short course of treatment appears to be a safe and acceptable option for patients with low back pain.

A meta-analysis of studies of acupuncture for osteoarthritis of the knee found that sham-controlled trials gave heterogeneous results as a consequence of variation in study design, and yielded clinically irrelevant short-term benefits. ${ }^{16}$ Wait-list controlled trials, however, suggested clinically relevant benefits of acupuncture in reducing pain.

A review evaluating the methodologic qualities and outcomes of 23 Japanese studies (controlled clinical trials) of acupuncture for conditions marked by chronic pain, such as back pain, neck pain, headache, and arthritis ${ }^{17}$ concluded that 17 of the studies were of low research quality, making it difficult to draw definitive conclusions from them. The review raised several important points, including the possibility that response to myofascial trigger points may be greater than the response to traditional acupuncture points in the treatment of chronic low-back pain. The Japanese studies also suggested that acupuncture may be more effective for tension type than for migraine headaches. ${ }^{17}$

Acupuncture may reduce cancer pain caused by tumor involvement or cancer treatment, and may also reduce the need for pain medication. ${ }^{18}$ Several studies have suggested that the paucity of rigorous, high-quality trials of acupuncture and cancer-related pain make it difficult to draw definitive conclusions about this, but that the trend toward benefit looks promising. ${ }^{19}$ One review suggests that acupuncture may not be as effective for treating cancer-related 
pain as it is for other pain-related conditions, and may be more helpful for other cancer-related symptoms, such as chemotherapyrelated nausea and vomiting. ${ }^{20}$

The literature reflects an increasing awareness of the need for more rigorous trials of acupuncture. There is also an overall trend toward a benefit of acupuncture for certain individuals with chronic pain. Unfortunately, many current studies of acupuncture and chronic pain state that no definitive conclusions can be drawn from current research, because this research suffers from methodologic flaws or other limitations such as small sample size.

Suggestions for future research include the need to know more about the optimal methods of acupuncture for specific conditions; an optimal schedule for treatments; and the need for long-term follow-up to assess the lasting effects of acupuncture.

\section{Massage}

The exact mechanisms by which massage may affect pain are not known, but it may help in managing chronic pain by directly relieving pain through muscle relaxation and indirectly through

$$
\begin{aligned}
& \text { Mind-body therapies may help reduce } \\
& \text { physical pain, nausea, and vomiting, and } \\
& \text { improve quality of life for people with cancer. }
\end{aligned}
$$

stress-reducing mechanisms such as the enhancement of parasympathetic nervous activity and activation of inhibitory mechanisms that suppress pain, increased relaxation, and improve sleep.

One review of massage in the management of chronic pain suggests strong support for massage use in chronic low-back pain, particularly in combination with exercise and education. ${ }^{21}$ The review found moderate support for massage in shoulder pain and for CranioSacral massage for tension headaches and mixed chronic pain conditions. The review provides preliminary support for the use of massage for carpal-tunnel syndrome and neck pain, but mixed results for massage's use in treating fibromyalgia. The review concludes that evidence varies for the benefits of massage in various conditions marked by chronic pain. However, the review makes the important observation that massage may not have an immediate effect on pain, but may show a significant effect when pain is assessed after several massage treatments and over time.

A recent report in the Annals of Internal Medicine, of nonpharmacologic options for the treatment of low back pain, found that $65 \%$ of primary-care physicians recommended massage therapy to the patient population with this condition. ${ }^{22}$ The authors observed that massage appears to be more effective for pain management when performed by trained massage therapists with many years of experience.
Massage may be useful in the management of cancer-related pain, but caution must be taken to ensure that massage is done by an experienced practitioner who is used to working with a population of patients with cancer, since massage can otherwise exacerbate cancer-related problems. ${ }^{18}$

Continued rigorous research on massage and pain should emphasize the importance of long-term follow up and the need for optimal treatment schedules. Methodologic flaws can make interpretation of the massage literature difficult, as can also the frequent use of massage in combination with other therapies and changes in lifestyle and behavior.

\section{Mind-Body Therapies}

Astin and colleagues, in a review of the effects of mind-body therapies (e.g., relaxation techniques, guided imagery, hypnosis, biofeedback, and cognitive behavioral therapy) for a variety of chronic pain conditions, drew the following conclusions: ${ }^{23}$

1. There is strong evidence that mind-body therapies have a moderately positive effect on low back pain as compared with the control of wait-listing for treatment.

2. Moderately strong evidence suggests that mind-body therapies may help reduce pain and physician visits in people with arthritis.

3. Strong evidence suggests that mind-body therapies may reduce headache frequency and symptoms, and that a combination of biofeedback and relaxation techniques may be the most effective complementary therapies for migraine and tension headaches

4. A variety of mind-body therapies may help reduce physical pain, nausea, and vomiting, and improve quality of life for people with cancer, although the evidence is stronger for this benefit in acute rather than in chronic pain

5. Findings for the benefits of mind-body therapies in fibromyalgia are equivocal.

Sierpina and colleagues have reviewed mind-body therapies for headache, and in a chart based on the evidence for a particular therapy and type of headache, ${ }^{24}$ give a variety of such therapies, including relaxation training, biofeedback, and cognitive therapy, an " $\mathrm{A}$ " rating-reflecting good-quality, patient-oriented evidence of its benefit-for treating migraine headache.

The Association of Applied Psychophysiology and Biofeedback has created an efficacy rating scale for the effect of biofeedback on a variety of conditions. ${ }^{25}$ In terms of conditions causing pain, the scale suggests that biofeedback is efficacious in treating headache and muscle-related TMJ disorders; probably efficacious in arthritis and chronic pain; and possibly efficacious in fibromyalgia.

\section{Hypnosis}

Hypnosis has been helpful in alleviating chronic pain caused by burns, cancer, rheumatoid arthritis, and other conditions in adults and children. One review suggests that hypnosis has been better than no treatment in decreasing pain and sometimes better than conventional treatment for a variety of pain-related conditions. ${ }^{26}$ The review states that the effects of hypnosis tend to be similar to 
those of progressive muscle relaxation and autogenic trainingboth of which include hypnosis-like suggestion. According to the review, few studies have compared hypnosis to placebo or minimally effective pain treatment, thus limiting the conclusions that can be drawn about the specific effectiveness of hypnosis. ${ }^{26}$

A review by Rogovik and colleagues indicates that in children, hypnosis may be particularly helpful for treating chronic headaches, bowel irritability, and cancer pain. ${ }^{27}$ The authors say that children are often easier to hypnotize and may respond better than adults to hypnosis for conditions of acute and chronic pain.

In a study of 53 children and adolescents ranging in age from 8 to 18 years and who had functional abdominal pain or irritable bowel syndrome, pain scores decreased significantly in groups randomly assigned to receive either gut-directed hypnotherapy or standard care $^{28}$ The hypnotherapy group went through six sessions over a period of 3 months, while the standard-care group received standard medical care for abdominal pain plus six sessions of supportive therapy. Hypnotherapy was superior to standard care in reducing pain scores. At 1 year, treatment was successful in $85 \%$ of the hypnotherapy group versus $25 \%$ of the standard-care group.

\section{Yoga}

Yoga may be useful for certain types of chronic pain. Because of its potentially rigorous physical nature, however, yoga also has the potential to be harmful in chronic pain. A high-quality study found that a 6-week program of Viniyoga yoga therapy was slightly better than exercise and moderately better than a self-care education book at 12 weeks of follow-up of patients with chronic low-back pain, and was superior to the self-care book at 24 weeks. ${ }^{29}$ At 24 weeks, $21 \%$ of yoga-treated patients reported use of medication, as compared with $50 \%$ in the exercise and self-care book groups.

\section{Expressive Writing}

Expressive writing has been found to reduce pain in patients with cancer and in women with chronic pelvic pain, as compared to controls who wrote about neutral topics. ${ }^{30}$ Expressive writing entails writing about a stressful or traumatic event for about 20 minutes on three or five occasions over consecutive days. Although expressive writing may also bring up distress, it has been found to improve outcomes for people with a variety of conditions including chronic pain. Benefits have included fewer stress-related visits to physicians; improved mood and affect; and fewer days of hospitalization. Expressive writing should be done in conjunction with a practitioner who is familiar with the process and can provide direction and support.

\section{Conclusions}

Working with psychologic and bodily tension is an important aspect of the management of chronic pain. Reducing stress and creating an improved sense of psychologic well-being may be as important in managing such pain as using treatments that work directly on the pain. Complementary therapies may be excellent means for working with this aspect of pain management.
A further key point is that an integrative approach to chronic pain must be tailored to the individual if it is to be successful. There is no universal remedy in the treatment of such pain. The greatest success appears to occur with treatment plans that incorporate: (1) conventional pain-management methods; (2) lifestyle and behavioral changes in the areas of stress reduction, exercise, and proper nutrition; (3) the appropriate use of complementary therapies; (4) and regularly scheduled physician visits to monitor progress. In my clinical experience, success is also improved by referring patients with chronic pain to highly experienced CAM practitioners who are specifically trained in the medical condition of concern.

A recent paper on CAM and pain makes the important point that understanding the approach of various CAM healing systems to pain, and the biologic mechanisms on which they are based, may also help the scientific understanding of pain and its treatment. ${ }^{31}$ Ancient healing traditions, such as Ayurveda or Traditional Chinese Medicine, utilize unique approaches and understanding in dealing with pain and correcting physical imbalances, and value long-term approaches rather than rapid remedies for managing chronic pain. These traditions also acknowledge the role of the mind and need for lifestyle and behavioral change in controlling pain. We would do well to draw on the knowledge of these systems when creating an integrated approach to pain.

Many research studies of CAM end on the note that research in the particular topic of the study is "flawed" and that "no definitive conclusions can be drawn" when interpreting study results. This is often true, but the body of research as a whole, and the experience of practitioners, physicians, and most importantly the people who use CAM clearly provides significant evidence for CAM's role and benefit in the integrative management of chronic pain. Chief among the needs for improving research on CAM in the management of chronic pain is the need for comparison studies of CAM modalities, studies of safety issues, and continued improvement in research on CAM and chronic pain.

\section{References}

1. Resources. Chronic Pain in America: Roadblocks to Relief. Conclusions. Size of the Chronic Pain Population. American Pain Society. Online document at: www.ampainsoc.org/links/roadblocks/conclude_road.htm Accessed February 13, 2008.

2. Cancer Facts and Figures 2007. Special Section: Cancer Related Pain. How Common is Pain in Cancer Patients? American Cancer Society. Online document at: www.cancer.org/downloads/STT/CAFF2007PWSecured.pdf Accessed February 13, 2008.

3. Resources. Chronic Pain in America: Roadblocks to Relief. Summary of Findings: Parameters of Moderate to Severe Chronic Pain. American Pain Society. Online document at: www.ampainsoc.org/links/roadblocks/sum mary1_road.htm Accessed February 13, 2008.

4. AAPM Facts and Figures on Pain. American Academy of Pain Medicine. Online document at: www.painmed.org/patient/facts.html\#pain Accessed February 14, 2008

5. Baliki MN, Geha PY, Apkarian AV, Chialvo DR. Beyond feeling: Chronic pain hurts the brain, disrupting the default mode network dynamics. J Neurosci 2008;28:1398-1403.

6. APS Glossary of Pain Terminology. American Pain Society. Online document at: www.ampainsoc.org/links / pain_glossary.htm Accessed February 13, 2008. 
7. National Center for Complementary and Alternative Medicine. The Use of Complementary and Alternative Medicine in the United States. Online document at: http://nccam.nih.gov/news/camsurvey_fs1.htm\#health Accessed February 18, 2008

8. Sawni A, Thomas R. Pediatricians' attitudes, experience and referral patterns regarding complementary/alternative medicine: A national survey. BMC Complement Altern Med 2007;7:18.

9. Tsao JC, Meldrum M, Kim SC, et al. Treatment preferences for CAM in children with chronic pain. Evid Based Complement Altern Med 2007;4:367-374. 10. Sherman KJ, Cherkin DC, Connelly MT, et al. Complementary and alternative medical therapies for low back pain: What treatments are patients willing to try? BMC Complement Altern Med 2004;4:9.

11. Cho ZH, Son YD, Han JY, et al. fMRI neurophysiological evidence of acupuncture mechanisms. Med Acupunct 2002;14:16-22.

12. Fredericson M, Pun S, Nelson L, Spiegel D. Medical acupuncture at a complementary medicine clinic: A 2-year retrospective study. Med Acupunct 2002;14:36-38.

13. Vickers AJ, Rees RW, Zollman CE, et al. Acupuncture of chronic headache disorders in primary care: Randomised controlled trial and economic analysis. Health Technol Assess 2004;8:iii,1-35.

14. Helms J. An Overview of Medical Acupuncture: Treatment uses of Medical Acupuncture. Medical Acupuncture. Online document at: www.medicala cupuncture.org/acu_info/articles/helmsarticle.html Accessed February 15, 2008.

15. Thomas KJ, MacPherson H, Thorpe L, et al. Randomised controlled trial of a short course of traditional acupuncture compared with usual care for persistent non-specific low back pain. BMJ 2006;333:623.

16. Manheimer E, Lind K, Lao L, et al. Meta-analysis: Acupuncture for osteoarthritis of the knee. Ann Intern Med 2007;146:868-877.

17. Itoh K, Kitakoji H. Acupuncture for chronic pain in Japan: A review. Evid Based Complement Alternat Med 2007;4:431-438.

18. Deng G, Cassileth BR. Integrative oncology: Complementary therapies for pain, anxiety and mood disturbance. CA Cancer J Clin 2005;55:109-116. 19. Bardia A, Barton DL, Prokop LJ, et al. Efficacy of complementary and alternative therapies in relieving cancer pain: A systematic review. J Clin Oncol 2006;24:5457-5464.

20. Conklin K. Acupuncture and the cancer patient. Med Acupunct 2002;14:23-32.
21. Tsao JC. Effectiveness of massage therapy for chronic, nonmalignant pain: A review. Evid Based Complement Alternat Med 2007;4:165-179.

22. Chou R, Huffman LH, American Pain Society, American College of Physicians. Nonpharmacologic therapies for acute and chronic low back pain: A review of the evidence for an American Pain Society/American College of Physicians clinical practice guideline. Ann Intern Med 2007;147:492-504.

23. Astin J, Shapiro SL, Eisenberg DM, Forys KL. Mind-body medicine: State of the science, implications for practice. J Am Board Fam Pract 2003:16:131147.

24. Sierpina V, Astin J, Giordano J. Mind body therapies for headache. Am Fam Physician 2007;76:1523-1524.

25. The Association of Applied Psychophysiology and Biofeedback. Efficacy. Online document at: www.aapb.org/i4a/pages/index.cfm?pageid=3336 Accessed February 15, 2008.

26. Jensen M, Patterson DR. Hypnotic treatment of chronic pain. J Behav Med 2006;29:95-124.

27. Rogovik AL, Goldman RD. Hypnosis for treatment of pain in children. Can Fam Physician 2007;53:823-825.

28. Vlieger AM, Menko-Frankenhuis C, Wolfkamp SC, et al. Hypnotherapy for children with functional abdominal pain or irritable bowel syndrome: A randomized controlled trial. Gastroenterology 2007;133:1430-1436.

29. Sherman KJ, Cherkin DC, Erro J, et al. Comparing yoga, exercise and a self-care book for chronic low back pain. Ann Intern Med 2005;143:849-856. 30. Baikie KA, Wilhelm K. Emotional and physical health benefits of expressive writing. Adv Psychiatr Treat 2005;11:338-346.

31. Hankey A. CAM and the phenomenology of pain. Evid Based Complement Alternat Med 2006;3:139-141.

Jane Hart, M.D., a board-certified internist, is a clinical instructor at Case Western Reserve University School of Medicine, Cleveland, Ohio, and was recently named Chair of the Committee on Integrative, Complementary and Alternative Medicine at Case Western Reserve School of Medicine.

To order reprints of this article, e-mail Karen Ballen at: Kballen@liebertpub.com or call at (914) 740-2100. 